Lastly, the opinion of those who have used the remedy seems to be that the dose should not exceed 1 c.c., at least for a long time. Excessive dosage seems to produce pain in the back, pain in the joints and pain in the wound.

In conclusion, I think we have in antituberculin a substance useful in the treatment of tuberculosis, in that it ameliorates certain symptoms seemingly associated with the toxic conditions present. While the exact value and action of the substance can not be proven by experiments upon guinea pigs, it seems to have been demonstrated clinically.

In closing this paper I must express my sincere thanks to Drs. J. C. Wilson and Kalteyer; Drs. James and.T. Miller Tyson; Dr. H. G. M. Kollock; Dr. E. S. Reynolds and J. C. Welch; Dr. James M. Anders; Dr. M. R. Ward; Dr. Guy Hinsdale, Dr. A. A. Eshner, Drs. J. and $\mathbf{S}$. Solis-Cohen, Dr. T. V. Crandall and others for their kind interest and aid in the investigation.

\section{EIGHTEEN YEARS OF PERSONAL OBSER- VATION OF TUBERCULOSIS IN ASHEVILLE, N. C.}

Presented to the Section on Practice of Medicine, at the Forty-eighth A mual Meeting of the American Medical Association held at Philadelphia, June 1-4, 1897.

BY JOHN HEY WILLIAMS, A.M., M.D

SURGEON-GENERAI N, C. N. G., ASHEVILLE, N.

In presenting this paper to your honorable body it is well to premise by stating that it is not my intention to exploit any new discovery or vaunt any new mode of treatment. I simply present deductions drawn from a personal observation of over two thousand cases, covering a period of eighteen years; during which time $I$ have been more or less actively engaged in the general practice of medicine in Asheville, N. C., and with, perhaps, special opportunities for such observaticn.

My excuse for this paper is, that I believe that the profession at large has a deep interest in such observations, made by one of a fair amount of intelligence and a deep interest in his profession. It is too often true that with the specialist the "wish is father to the thought," and conclusions are reached long before the clinical facts will justify such conclusions. It is not my intention to burden this paper with futile speculations and reports of cases.

For the last thirty years or more, the plateau of western North Carolina, of which Asheville is the center, has been the Mecca toward which the faces of many of our afflicted have been turned.

Eighteen years ago I was myself an invalid, suffering from supposed pulmonary tuberculosis-I say supposed, having then no means of positive diagnosis. I was advised by such eminent authorities as Da Costa, Weir Mitchell and the lamented Pancoast that I could not live a year longer in Philadelphia and that a change of climate was necessary. My attention was drawn to western North Carolina by the writings of Hon. E. J. Aston, to whose memory I would that time and ability would permit a more fitting tribute, and the reports of Dr. J. W. Gleitsman, now of New York, who was then conducting a sanitarium for pulmonary diseases at Asheville. To his scientific observation is due much of our knowledge of the climatologic advantages of this spot. At that time Asheville boasted of but 1,700 people, thoroughly imbued with the old time southern hospitality, and pride in their beautiful country. A few month' residence there, and a more thorough knowledge of its climatic attributes, decided me that to obtain a perfect cure I should take up a more prolonged residence. I decided to remain five years at least. With passing years came confirmed health, and I have remained eighteen, with pleasure and profit. With returning health I devoted myself to the practice of medicine, but as time passed the increasing number of invalids who sought our hospitable doors caused my work to run more largely in to the line of tubercular troubles. Prior to the tremendous discovery of Koch the treatment of such cases was in no manner specialized. Few if any notes were made of the treatment and progress of the cases. Systematic record of temperature, pulse and respiration was seldom made and it was not until after the discovery of the tubercle bacilli and the necessary association of their presence with the usual phenomena associated with consumption, as we now understand it, that a sharp interest was taken in such cases and the work of our confrères all over the world was closely scrutinized, and independent investigations set on foot with the hope that we might add something to our armamentarium. With greater precision in diagnosis due to improved aids and the closer attention of the profession at large, the earlier diagnosis of tuberculosis was possible.

The influence of climate in the therapy of the disease was given closer attention and its influence in the arrest and cure of the disease has been from time to time strongly set forth by all of our leading specialists, not only to the profession but to the laity as well. The influence of all this teaching is that yearly still larger numbers of primary cases are seeking the health-giving plateau of western North Carolina and western Kansas and Colorado. The ease of access-less than twenty-four hours from New York and only eighteen from the Ohio Valley, with its now well-known climatic advantages, has made this favored region the choice of most physicians of the North and East.

With a proper understanding of all that climate can do for such cases has come the full knowledge that climate is not all, and we have learned, too, that "change of climate" in part consists of a complete change in the environment of the patient, which is so large a factor in the health-restoring attributes of most of our health resorts. The freedom from business cares, household and social duties, is by no means to be underrated. Then comes, too, the change in all the nutritive surroundings of the patient; the bracing air, surcharged with ozone as shown by the meteorologic bureau, the cloudless skies and magnificent scenery, where external impressions count for so much, all conduce to an active out-door life. To such as can and will lead such a life, climate means much-those who can not and will not submit to the intelligent discretion of the painstaking physician and put off having a "good time," till the restoration of their health, had better stay at home. The result in such cases will bring discredit upon the remedy. The care and supervision of the physician whose intelligence and skill has been or should be vastly improved by the observation of the large number of cases that yearly pass in review before him, is necessary in regulating the daily details of the patient's life.

With the closer attention that has of late years been given to the prodromic signs of latent tuberculosis 
comes an earlier removal from the environment of the patient with corresponding benefit.

In speaking of latent tuberculosis, I have had im. pressed on me the influence of heredity and a careful study of over 70 per cent. of my patients shows the hereditary taint. In this connection comes the thought of prophylaxis. I would strongly urge the propriety and even necessity of the family physician making regular and stated examinations of every member of the families who may be intrusted to his care, in whom there is even a remote suspicion of the hereditary taint. The importance of the early recognition of tuberculosis is being daily urged upon usso much can be done in the earlier stages and so little in the later. Cases in the later stages of the disease, with large suppurative cavities, septic chills and fevers, amyloid degeneration of the kidneys, tubercular infection of the intestinal tract with diarrhea-any or all of these I have seen on their arrival in Asheville and have had to advise their immediate returncan have the pathway to the tomb made much smoother with the comforts of home.

The various treatments that have been exploited by their fond discoverers have all passed in kaleidoscopic review, Bergeon, superheated air, etc. Of the most of them it is unnecessary to speak. I have had an opportunity to observe all of them as well as to experiment with the various remedies that have found favor with the profession.

The grave disturbances of nutrition in all cases of tuberculosis, whether it be primary or secondary, is a matter of deep interest. It is a recognised fact that only through suitable changes in all the nutritive processes and surroundings of the patient can we hope to deal successfully with the profound cachexia of tuberculosis in any form.

Possessing no known specin̂c, we have been led to experiment with any and all remedies, foods and physical surroundings that contribute in any way to the building up of the physical and vital forces. "The gamut has been run in the way of chemic foods-socalled-but we have to admit that the chemic laboratory has not as yet furnished us with anything that can supplant nature in the catabolic and metabolic processes necessary to a stable equilibrium in the physical economy. Anything that will assist nature to resume a wholesome and healthy function of digestion and assimilation will prove to be a valuable addition to our armamentarium.

Intestinal antisepsis-thorough and complete as may be-appears to have first place in conducing to the nutrition of the patient. In this class of remedies creosote appears to have first rank. I have observed no special benefit from the inordinately large doses given by some. I have given it in doses of from 20 to 90 minims daily, but have decided that I get as good results from the smaller as from the larger doses.

Next to creosote, and I am sometimes of the opinion that it should come first, is the internal administration of ichthyol. I have used the remedy for the past eight years in daily doses of from 20 to 100 minims, and my case books show a very material modification of the symptoms in a large percentage of cases, especially marked in the earlier stages. Glandular cases and those showing evidence of gastric and duodenal catarrh come especially within the range of of its power. Since reading the remarkable results obtained by Scarpa I have had my faith in its powers much strengthened. In no one case have I noticed any unfavorable result from its usage, except an occasional bronzing of the skin, coming on about the third or fourth week of its exhibition and lasting a week or two. In a large percentage of the cases in which I have used it I have noticed a gradual involution and degeneration of the bacilli, with a reduction in number, and their final disappearance. The assimilation is improved and the patient takes on flesh; the character of the sputum is changed in a marked manner, loosing its heavy, viscid, nummular character and becoming thinner, with greater ease in expectoration. There is an improvement in the appetite and digestion with recession of the daily temperature and lessened night sweats.

The use of the pneumatic cabinet with forced inhalation of the vapors of creosote, oil of pine needles, oil of cedar and the various balsamic preparations finds great favor. The stimulation of the local circulation gives consequent freer oxygenation of the blood. The expansion of the bronchioles and vesicles helps to free them from retained mucus and gives a larger breathing capacity, and it counteracts the contraction of connective tissue from inflammatory deposits.

I think it is the general consensus of medical opinion that so-called intra-pulmonary medication, whether by sprays, vapor or steam baths, is, per se, of but little avail and that our best efforts should be directed to the restoration of the impaired nutrition and the stimulation of the leucocytes and phagocytes of the blood. If the theory of Metchnikoff be true, and it has not yet been discredited, the remedies that will stimulate this vital action will prove of best service to us.

In this connection I will mention my personal use of Koch lymph and later of the modified product of Klebs known as Antiphthisin.

I was an early user of Koch's tuberculin and was, as were most of us, enthusiastic over the promises of the future. Unfortunate results in some of my earlier cases, however, produced such revulsion of feeling that for months the remedy was allowed to fall into innocuous desuetude. More careful study, however, of the physiologic action of the remedy, with more favorable reports from observers at other points, induced me to give it further trial with careful precaution as to the initial dosage and thorough espionage of the patient for several hours after its administration. With care in its usage, I secured in a few cases some remarkably favorable results, but in the majority of cases confess to disappointment. The advent of the serum therapy gave renewed interest in the investigations of Koch and Klebs. The modification of the remedy as proposed under the name of antiphthisin or purified tuberculin seemed to offer a safer remedy than the earlier product of Koch's laboratory and to admit of a more extended application. It has been, I think, proven by clinical observation and experience that in the earlier or prodromic stages the antiphthisin Klebs or the later product of Koch's laboratory may give almost marvelous results. My case books show that within the last two years I have had not less than thirty absolute and unequivocal recoveries under the administration of this remedy alone.

It is not within the province of this paper to discuss the physiologic action of this remedy. I am simply noting clinical results. I may, however, mention that I do not think that antiphthisin possesses 
any bactericidal power as does the antitoxin of diphtheria but that it does produce in the body of the patient, by some catalytic power acting as a special ferment upon the healthy tissues of the body, that stimulation of phagocytosis that enables the patient to deal with the bacilli, producing if you please a personal antitoxin. With such specific treatment of the patient comes the general care of all the functions of the body. Septic chills and fevers are not to be controlled by quinin or the coal-tar products. The usage of these latter remedies has been generally discarded. The relaxed skin renders the patient more susceptible to the effect of the diurnal changes common to all elevated latitudes. The daily rise of temperature is best combated with perfect rest in bed with liquid food. With extreme temperature, I have gotten the best results from the use of the ice-bag over the precordium or cold packs to the chest. Oil of turpentine with subgallate of bismuth appears to give the best results in diarrheic cases-opiates are seldom required, save possibly in the form of codein to control cough. The large diurnal range of the thermometer renders the wearing of woolen or silk or other non-conducting material next to the skin imperative. The powerful and stimulating effect of a strychnia nitrate upon the vascular and respiratory centers makes it a valuable adjunct.

Hematic remedies, especially Gude's pepto-mangan, gives excellent results, epescially in cases of an anemic character and in those of a mixed infection. My experience in the Shurly-Gibbs treatment in sixty-four cases has been unsatisfactory in every respect. The Edson aseptolin has proven to be of service in about 14 per cent. of those cases that came to me with a his. tory of malarial infection.

The ingestion of as large quantities of nitrogenous foods as can comfortably be taken care of by the digestive organs, the out-door life, the careful conservation of all the forces of the body will add much to that uncertain factor known as climate. In the selection of cases suitable for transfer to the plateau of western North Carolina I have a few words of caution to offer. Examine your cases carefully with all the aids that modern research has given us. A microscopic examination of the sputum will be of invaluable aid. Cases of mixed infection of the mesentery and the great eliminative organs are by far better off at home. The familiar surroundings, the faith and hope inspired by the old and trusted family physician, the thousand nameless comforts of home will prolong a possibly useful life that would succumb certainly and speedily under the depressing influence of absence from home and friends. Nostalgia is as important a factor in these as with our sick and wounded in the late war.

I have noticed but little effect upon the nervous system from our medium altitude, except that in most cases it has a distinctly soothing effect and that our visitors sleep better here than at home. Cases of a hemoptistic character are usually advised to try the lower altitudes of the eastern and southern slope of the Blue Ridge and I think that Tryon, in the western extremity of the famous Thermal Belt, is the ideal spot.

The financial condition of the patient should also be carefully considered, since to get the best results from climatic influences he should be provided with not only the necessaries but even the comforts and luxuries of life. Very little benefit can be obtained from such climate as can be obtained from a little back room in some second- or third-class boarding house. Let him keep away from the clubs and hotel lobbies and abstain from all the frivolities that are found in all well-known resorts. Do not advise him that the climate will do all for him that is necessary. Advise him by all means to consult on his arrival one who is familiar with all the various things that will conduce to his well being and capable of advising against the dangers that may result from a total change in his habits and surroundings. You will find more than one physician at all such resorts and it is presumable that they are as honest as the general run of the profession.

I would sugggest that you submit your observation of the patient, with history of prior treatment to the physician to whom you may intrust him, noting the remedies which you have found of special benefit, and any peculiarity of temperament or habit with which you may be acquainted. Above all be honest with your patient. It is not kindness to tell him that he has a little bronchial trouble, just a little catarrh or something of that sort, and that a few days' sojourn in a pleasant climate will set him all right. Tell him why you send him and the necessity of devoting all his time and energy to getting well. Tell him the truth.

53 Haywood Street.

\section{DR. DELANCEY ROCHESTER'S "REPORT ON THE TREATMENT OF PULMONARY TUBERCULOSIS."-REPLY.}

BY PAUL PAQUIN, M.D. st. Lovis, Mo.

Under the above caption appears in the JournaL of the American Medical Association, July 10, 1897, the report of Dr. DeLancey Rochester of Buffalo, N.Y., as presented to the Section on Practice of Medicine, at the Forty-eighth Annual Meeting of the American Medical Association, held at Philadelphia, June 1-4, 1897. This report I had the pleasure of hearing and was disappointed in not being able to discuss it, owing to the numerous other papers on tuberculosis presented before that and other Sections of the Association, and for the discussion of which only five minutes were granted to each member.

The report of Dr. Rochester seems to be a comparative statement of the results he obtained in the use of Edson's aseptolin, Paquin's tubercle antitoxin and Vaughan's nuclein. I have read it carefully and am compelled to reply because of the manifest injustice it does the cause of sero-therapy and myself. In order to present my case properly before the readers of the Journal and the members of the AMERIcan Medical Association, it is necessary for me to give a history of Dr. Rochester's tests of "Puquin's" serum, and for this purpose I will be obliged, much to my regret, to quote from his letters to me and from mine to him. I feel justified in doing this because Dr. Rochester in his conclusions, gained from such a meager experience, declares against Paquin's tubercle antitoxin in unmeasured terms, and because the language and tone throughout the report is one to discourage the profession from using serum, and to belittle the method of treatment, to class it with aseptolin, and to exalt, beyond reason, another therapeutic agent. Dr. Rochester's grounds are not tenable.

On Nov. 28, 1896, Dr. Rochester addressed the lab. 